\title{
Inhibition of the VEGF signaling pathway attenuates tumor-associated macrophage activity in liver cancer
}

\author{
SHOHEI OKIKAWA, YUJI MORINE, YU SAITO, SHINICHIRO YAMADA, KAZUNORI TOKUDA, \\ HIROKI TERAOKU, KATSUKI MIYAZAKI, SHOKO YAMASHITA, TETSUYA IKEMOTO, \\ SATORU IMURA and MITSUO SHIMADA
}

Department of Surgery, Institute of Biomedical Sciences, Tokushima University Graduate School, Tokushima 770-8503, Japan

Received August 19, 2021; Accepted January 13, 2022

DOI: $10.3892 / o r .2022 .8282$

\begin{abstract}
Tumor-associated macrophage (TAMs) are paramount for tumor progression and immune tolerance in the tumor microenvironment of various types of cancer, including liver cancer. The aim of the present study was to investigate the effect of vascular endothelial growth factor (VEGF) inhibition on TAM polarization and function during their interactions with macrophages and liver cancer cells. TAMs were induced by culturing M0 macrophages with cancer cell-conditioned medium. TAMs cultured with cancer cell-conditioned medium and vascular endothelial growth factor (VEGF) inhibitor were defined as modified TAMs, and the expression levels of TAM-associated markers and VEGF receptor 2 were evaluated using reverse transcription-quantitative polymerase chain reaction (RT-qPCR). The effects of TAMs and modified TAMs on cancer cell proliferation and migration were investigated using conditioned medium. Programmed death-ligand 1 (PD-L1) mRNA expression in modified TAMs and cancer cells cultured in modified TAM-conditioned medium (TAM-CM) for $48 \mathrm{~h}$ was examined using RT-qPCR. In order to investigate signaling pathways in macrophages, western blot analysis was performed. CD163 and CD206 and M2 macrophage marker expression was upregulated in TAMs and modified TAMs. Modified TAM-CM exhibited a decreased ability to
\end{abstract}

Correspondence to: Dr Yuji Morine, Department of Surgery, Institute of Biomedical Sciences, Tokushima University Graduate School, 3-18-15 Kuramoto-cho, Tokushima 770-8503, Japan E-mail: ymorine@tokushima-u.ac.jp

Abbreviations: CCL2, C-C motif chemokine ligand 2; CM, conditioned medium; EMT, epithelial-mesenchymal transition; IL, interleukin; Nrf2, nuclear factor (erythroid-derived 2)-like 2; PMA, phorbol 12-myristate 13-acetate; PD-L1, programmed death-ligand 1; TAM, tumor-associated macrophage; TME, tumor microenvironment; VEGF, vascular endothelial growth factor; VEGFR, vascular endothelial growth factor receptor

Key words: liver cancer, VEGF, tumor-associated macrophages, PD-L1, tumor microenvironment promote cancer cell proliferation and migration in comparison with the use of TAM-CM. The VEGF concentration was significantly higher in the TAMs than in M0 macrophages; however, the modified TAMs displayed a significantly lower VEGF secretion than TAMs. PD-L1 expression was decreased in modified TAMs as compared with TAMs. Western blot analysis revealed that the Akt/mTOR signaling pathway was significantly suppressed in the modified TAMs compared with TAMs. It was observed that TAMs cultured in a VEGF-depleted environment displayed lower secretion levels of cytokines involved in tumor progression and a decreased immune tolerance-inducing ability. On the whole, the results of the present study suggested that VEGF inhibition in TAMs may be a potential therapeutic target for liver cancer.

\section{Introduction}

The tumor microenvironment (TME) plays a crucial role in cancer occurrence and progression. The TME consists of endothelial cells, fibroblasts, vascular components, macrophages, immune cells and secreted cytokines $(1,2)$. Targeting the TME represents a potential approach for the treatment of cancer at the tumor and cellular level simultaneously.

Tumor-associated macrophages (TAMs) are one of the most common populations of tumor-infiltrating immune cells in the TME (3). Macrophages can be classified into two subtypes, the M1 phenotype (classically activated) and M2 phenotype (alternatively activated). TAMs are usually polarized toward the tumor-promoting M2 phenotype rather than the tumoricidal M1 phenotype (4). Several studies have demonstrated that high numbers of TAMs in the TME are associated with a poor prognosis in various types of cancer, including ovarian, pancreatic and lung cancer (5-8). Through the secretion of various cytokines, TAMs can promote tumor growth, angiogenesis and epithelial-mesenchymal transition (EMT) and reduce immune cell antitumor activity, leading to the suppression of antitumor immune responses in the TME (9-11).

Vascular endothelial growth factor (VEGF) is an important cytokine in the TME, and cancer cells and TAMs are the major sources of VEGF $(12,13)$. During tumor progression, VEGF secreted by cancer cells binds to the VEGF receptor 
(VEGFR) of endothelial cells, thereby enhancing endothelial cell proliferation and migration, and promoting angiogenesis near the tumor site (14). Furthermore, it has been reported that VEGF secreted by cancer cells functions in an autocrine and paracrine manner, and enhances tumor growth via the activation of the VEGF/VEGFR signaling pathway in cancer cells (15). Cancer cells have been also reported to produce cytokines and chemokines, such as interleukin (IL)-4, IL-10, and $\mathrm{C}-\mathrm{C}$ motif chemokine ligand 2 (CCL2) to induce TAM infiltration. CCL2 is involved in the recruitment of monocytes, IL-4 and IL-10, polarizing monocytes toward the M2 phenotype (termed TAMs) (16). TAMs that are recruited and activated by cancer cells have been reported to secrete VEGF and promote tumor progression (17). It has been previously reported by the authors that VEGF secreted by TAMs may promote the EMT of cancer cells via transcription factor nuclear factor (erythroid-derived 2)-like 2 (Nrf2) activation in cancer cells (18). However, the findings of the effects of VEGF on macrophages have thus far been controversial $(19,20)$. Wheeler et al (19) reported that VEGF treatment significantly enhanced the upreglation of M2 markers of macrophages, while Linde et al (20) reported that VEGF was not involved in the M2 polarization of macrophages in vitro. Thus, the effects of VEGF secreted by cancer cells on TAM polarization remain unclear.

Programmed death-ligand 1 (PD-L1) plays a crucial role in the TME by suppressing the anti-tumor T cell-mediated immune response. Novel therapies targeting the PD-L1/programmed cell death protein 1 axis have been developed for cancer treatment. In the treatment of liver cancer, bevacizumab (a VEGF-A inhibitor) and atezolizumab (a PD-L1 inhibitor) have already been clinically applied as cancer treatments $(21,22)$. Previous studies have suggested that the tumor stroma, including TAMs, is a regulator of PD-L1 expression in cancer cells (1-3). However, whether VEGF secreted by TAMs is involved in PD-L1 regulation in the TME remains unclear.

In the present study, the role of VEGF in the TME of liver cancer was investigated with particular focus on the effects of VEGF inhibition on TAM function. It was observed that a VEGF-depleted environment attenuated the tumor-promoting function of TAMs and the ability of TAMs to enhance PD-L1 expression in cancer cells through the decreased cytokine secretion of TAMs, and via the inactivation of the VEGFR2/Akt/mTOR pathway.

\section{Materials and methods}

Cells and cell culture. Huh-7 (RCB1366) and HepG2 (RCB1648) cells (human liver cancer cell lines) were obtained from the RIKEN BioResource Center Cell Bank. THP-1 cells (a human monocyte cell line) were obtained from the Culture Collections of Public Health England (https://www. phe-culturecollections.org.uk/). The Huh-7 and HepG2 cells were maintained in DMEM (Thermo Fisher Scientific, Inc.), and the THP-1 cells were maintained in RPMI-1640 (Wako Pure Chemical Industries, Ltd.). Media were supplemented with 10\% FBS (Thermo Fisher Scientific, Inc.) and 1\% penicillin/streptomycin (Gibco; Thermo Fisher Scientific, Inc.). To induce cell differentiation into M0 macrophages, the THP-1 cells were exposed to $150 \mathrm{nmol} / \mathrm{l}$ phorbol 12 -myristate 13-acetate (PMA; MilliporeSigma) for $48 \mathrm{~h}$. All cells were maintained under a $5 \% \mathrm{CO}_{2}$ atmosphere at $37^{\circ} \mathrm{C}$.

Preparation of conditioned medium (CM) and TAMs. The Huh-7 and HepG2 cells were cultured to $80 \%$ confluency in 100-mm culture dishes, in order to obtain CM. All cells were washed with pre-warmed PBS twice and then incubated with fresh medium without FBS. Following $48 \mathrm{~h}$ of incubation at $37^{\circ} \mathrm{C}$, the supernatant was collected, centrifuged at $800 \mathrm{x} \mathrm{g}$ for $5 \mathrm{~min}$ at $22^{\circ} \mathrm{C}$, and filtered through a $0.2-\mu \mathrm{m}$ sterile filter. The cancer cell-derived CM (Huh-7-CM and HepG2-CM) was used without additional FBS.

To obtain TAMs, M0 macrophages were treated with cancer cell-derived CM (Huh-7-CM and HepG2-CM). CM was added to the culture medium at a ratio of $1: 1$, and the cells were stimulated for $48 \mathrm{~h}$ at $37^{\circ} \mathrm{C}$. TAMs induced from Huh-7-CM were defined as TAM(Huh7), and TAMs induced from HepG2-CM were defined as TAM(HepG2). To obtain modified TAMs, M0 macrophages were treated with cancer cell-derived CM containing VEGF antibody. VEGF antibody (cat. no. MAB293; R\&D Systems, Inc.) was added to the medium at the concentration of $60 \mathrm{ng} / \mathrm{ml}$.

The M0-CM, TAM-CM and modified TAM-CM were collected from the M0 macrophage culture, TAM culture and modified TAM culture, respectively, in the same manner as described above. In the proliferation and migration assays, the CMs were added to the culture medium at a ratio of 1:1. The same concentration of human IgG (cat. no. 1-001-A; $R \& D$ Systems, Inc.) was used as a control.

Cell proliferation assay. Cell Counting Kit-8 (CCK-8) assay (Dojindo Molecular Technologies, Inc.) was performed in accordance with the manufacturer's protocol. Briefly, $10 \%$ CCK-8 reagent was added to each well. The plates were incubated at $37^{\circ} \mathrm{C}$ for $2 \mathrm{~h}$, and the absorbance was analyzed at $450 \mathrm{~nm}$ using a microplate reader (SpectraMax i3; Molecular Devices, LLC).

Migration assay. Transwell migration assays were performed using 24-well plates with $8-\mu \mathrm{m}$ pore membrane inserts (Corning, Inc.) in accordance with the manufacturer's protocol. Huh-7 and HepG2 cells were seeded in the upper chamber at a concentration of 20,000 cells in $100 \mu \mathrm{l}$ of medium containing $1 \%$ FBS. In the lower chamber, corresponding CM was added to the 10\% FBS DMEM culture medium at a ratio of 1:1; the final FBS concentration was 5\%. Following $24 \mathrm{~h}$ of incubation at $37^{\circ} \mathrm{C}$, cells that had migrated to the bottom of the Transwell membrane were fixed with $4 \%$ paraformaldehyde for $15 \mathrm{~min}$. The membrane was stained using $0.2 \%$ crystal violet solution (Wako Pure Chemical Industries, Ltd.) for $20 \mathrm{~min}$ at room temperature, and stained cells were counted using a phase-contrast microscope (BX43; Olympus Corporation) in three random fields per membrane (x200 magnification).

Wound healing assay. Huh-7 and HepG2 cells were seeded in six-well plates and grown to $90 \%$ confluency. The cell monolayer was scratched using a plastic pipette tip across the well to create a 1-mm-wide gap. The detached cells were removed by washing with PBS twice. The well was then replenished with fresh DMEM medium containing 1\% FBS followed by 
the addition of corresponding $\mathrm{CM}$ at a ratio of $1: 1$, the final FBS concentration was $0.5 \%$, the cancer cells were cultured for a further $24 \mathrm{~h}$ at $37^{\circ} \mathrm{C}$. Images of the wound areas were captured using a phase-contrast microscope (magnification, x40; DP22-CU; Olympus Corporation) at 0 and $24 \mathrm{~h}$ after scratching. The wound healing rates were calculated using Image J v1.46r software (National Institutes of Health) and using the following equation: Wound healing rate $(\%)=[$ area $(0 \mathrm{~h})$-area $(24 \mathrm{~h})]$ /area $(0 \mathrm{~h}) \times 100$.

Cytokine array. Cytokines in M0-CM and TAM-CM were detected using a Proteome Profiler Human Cytokine Array kit (cat. no. ARY005B; R\&D Systems, Inc.) following the manufacturer's protocol. The chemiluminescence signal intensities on the membranes were detected using a Lumino Image Analyzer (Amersham Imager; Cytiva).

ELISA. The concentrations of VEGF and MMP-9 in the CM were detected using a VEGF ELISA kit (cat. no. DVE00; R\&D Systems, Inc.) and an MMP-9 ELISA kit (cat. no. DMP900; R\&D Systems, Inc.), respectively, following the manufacturer's protocol. The absorbance was measured at $450 \mathrm{~nm}$ using a microplate reader (SpectraMax i3; Molecular Devices, LLC).

Reverse transcription-quantitative polymerase chain reaction (RT-qPCR). The RNeasy Mini Kit (Qiagen $\mathrm{GmbH}$ ) was used to extract total RNA from the cells following the manufacturer's instructions. The total RNA concentration was determined using a spectrophotometer (NanoDrop 2000; Thermo Fisher Scientific, Inc.) according to manufacturer's instructions. cDNA was synthesized from $2.5 \mu \mathrm{g}$ total RNA using a High-Capacity cDNA Reverse Transcription kit (4368813, Applied Biosystems; Thermo Fisher Scientific Inc.) in a final volume of $50 \mu \mathrm{l}$. The cycling conditions for the reverse transcription were as follows: Incubation at $25^{\circ} \mathrm{C}$ for $10 \mathrm{~min}, 37^{\circ} \mathrm{C}$ for $120 \mathrm{~min}, 85^{\circ} \mathrm{C}$ for $5 \mathrm{~min}$. All samples were kept at $-20^{\circ} \mathrm{C}$ until ready for use. The StepOnePlus Real-Time PCR System (Applied Biosystems; Thermo Fisher Scientific Inc.) was used to conduct qPCR. The qPCR conditions consisted of an Initial denaturation for $3 \mathrm{~min}$ at $95^{\circ} \mathrm{C}$, followed by 40 cycles of $30 \mathrm{sec}$ denaturation at $95^{\circ} \mathrm{C}$, annealing for $30 \mathrm{sec}$ at $58^{\circ} \mathrm{C}$ and extension at $72^{\circ} \mathrm{C}$ for $45 \mathrm{sec}$. The final extension was carried out at $72^{\circ} \mathrm{C}$ for $10 \mathrm{~min}$. The primers from TaqMan assays (assay identification number) used in the present study are as follows: CD163 (Hs00174705_m1),CD206 (Hs00267207_m1), VEGFR2 (Hs00911700_m1) and PD-L1 (Hs00204257_m1). GAPDH (4326317E) was used as an internal control. All primers were purchased from Thermo Fisher Scientific, Inc. The data were analyzed using the $2^{-\Delta \Delta \mathrm{Cq}}$ method (23). The results are presented as the fold changes of the relative mRNA expression for each experimental group compared with that in the control group.

Western blot analysis. Cell lysates were obtained, and western blot analysis was performed as previously described $(18,24)$. Briefly, total proteins were extracted by lysing the cells using RIPA lysis buffer (Thermo Fisher Scientific, Inc.), containing a protease inhibitor cocktail (Sigma-Aldrich; Merck KGaA) and a PhosSTOP phosphatase inhibitor cocktail (Roche Diagnostics). The protein concentration was measured using a
BCA kit (Thermo Fisher Scientific Inc.). Equal amounts $(20 \mu \mathrm{g})$ of extracted proteins were separated on 10\% SDS-PAGE gels and transferred onto PVDF membranes (Bio-Rad Laboratories, Inc.). The membranes were incubated with the indicated primary antibody overnight at $4^{\circ} \mathrm{C}$. The membranes were then incubated with appropriate HRP-conjugated secondary antibody for $1 \mathrm{~h}$ at room temperature. The proteins were detected using ECL reagents (Cytiva). Western blot densitometry band quantification was performed using ImageJ v1.46r software (National Institutes of Health). The primary antibodies used in the present study, along with the corresponding dilutions, are listed in Table I. The p-Akt antibody used in the present study detected endogenous Akt phosphorylated at Ser473.

Statistical analysis. All statistical analyses were performed using JMP software (version 13; SAS Campus Drive). The Student's t-test was used for statistical comparisons between two groups, and one-way ANOVA with the Tukey-Kramer test was used for statistical comparisons among three or more groups. All experiments were repeated more than three times. Data are expressed as the mean $\pm \mathrm{SD}$. A P-value $<0.05$ was considered to indicate a statistically significant difference.

\section{Results}

Polarization of MO macrophages toward the M2 phenotype (termed TAMs) by cancer cell-CM. To generate TAMs, THP-1 cells were first stimulated with PMA for $48 \mathrm{~h}$ to induce their differentiation into M0 macrophages. The M0 macrophages were then cultured with Huh-7-CM or HepG2-CM for a further $48 \mathrm{~h}$ (Fig. S1A). The two generated TAM lines, TAM(Huh-7) and TAM(HepG2), expressed increased mRNA levels of the M2 macrophage markers, CD163 and CD206, as compared with the expression levels in M0 macrophages $(\mathrm{P}<0.05$, Fig. S1B).

TAMs enhance liver cancer cell line proliferation and migration. Subsequently, the effects of TAMs on the malignant potential of Huh-7 and HepG2 cells were investigated. TAMs were established using CM from Huh-7 and HepG2 cells; CM from the two sets of TAMs was then collected, and the Huh-7 and HepG2 cells were cultured with the TAM-CM (Fig. S2A). TAM-CM from both cell lines significantly increased the proliferation and migration of the Huh-7 and HepG2 cells compared with the effects of $\mathrm{M} 0-\mathrm{CM}(\mathrm{P}<0.05$, Fig. S2B-D). These results indicated that secreted factors from TAMs may play a crucial role in the effects of TAMs on the proliferation and migration of cancer cells.

Inhibition of VEGF secretion by TAMs attenuates liver cancer cell proliferation and migration. A cytokine array using TAM(Huh-7)-CM was used to compare the differences in cytokine secretion between TAMs and M0 macrophages. The results illustrated that TAMs secreted increased levels of VEGF in comparison with the M0 macrophages (Fig. 1A). The VEGF concentrations in the TAM(Huh-7)-CM and TAM(HepG2)-CM were then examined using ELISA. The results revealed that both TAM cell lines secreted significantly higher VEGF levels than the M0 macrophages $(\mathrm{P}<0.001$, Fig. 1B). To investigate the effects of VEGF secretion by TAMs 
Table I. Details of antibody sources and concentrations used for western blot analysis.

\begin{tabular}{|c|c|c|c|}
\hline Antibody & Company & Cat. no. & Dilution \\
\hline Akt (total-Akt) mAb & Cell Signaling Technology, Inc. & $4691 S$ & $1: 1,000$ \\
\hline Phospho-Akt (p-Akt) mAb & Cell Signaling Technology, Inc. & $4060 \mathrm{~S}$ & $1: 1,000$ \\
\hline mTOR (total-mTOR) mAb & Abcam & ab32028 & $1: 1,000$ \\
\hline Phospho-mTOR (p-mTOR) mAb & Abcam & ab109268 & $1: 1,000$ \\
\hline$\beta$-actin mAb & Cell Signaling Technology, Inc. & $4970 \mathrm{~S}$ & $1: 1,000$ \\
\hline HRP-linked anti-rabbit antibody & Cell Signaling Technology, Inc. & $7074 \mathrm{~S}$ & $1: 3,000$ \\
\hline
\end{tabular}

mAb, monoclonal antibody.

A
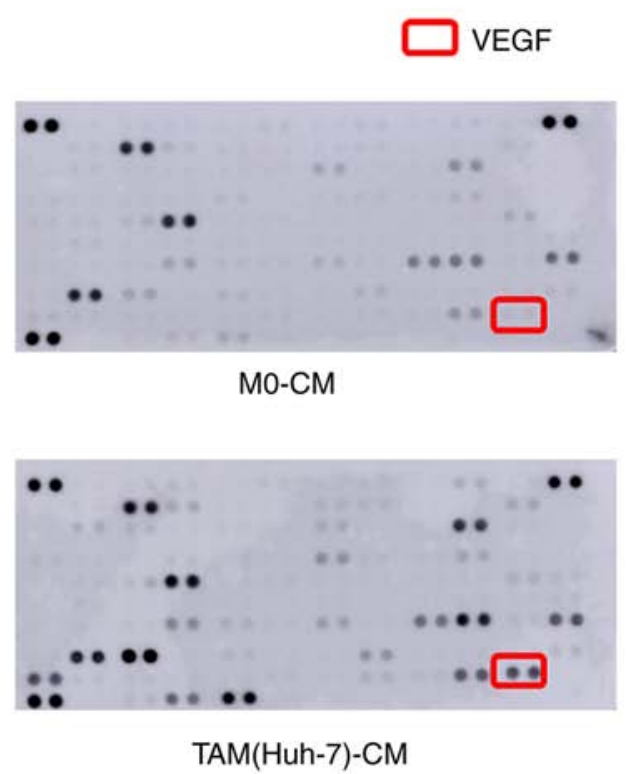

B

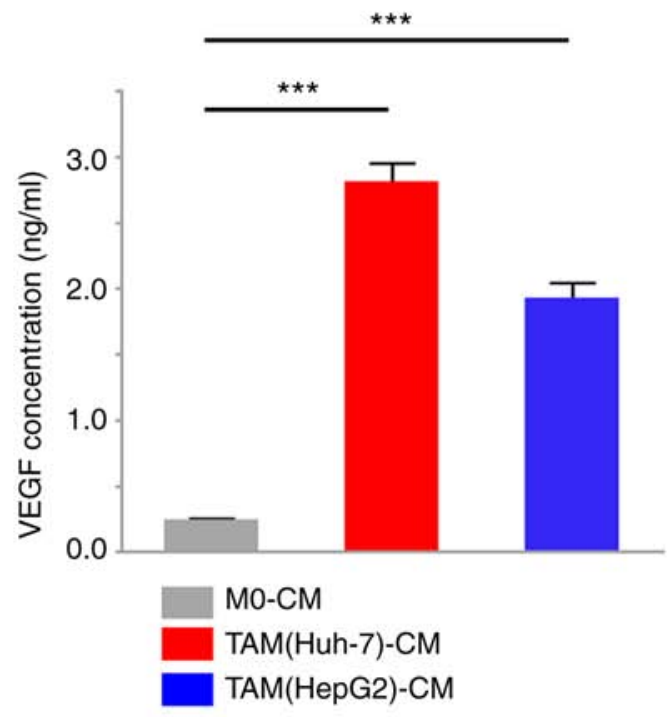

Figure 1. TAMs secrete high amounts of VEGF. (A) Cytokine arrays of M0-CM and TAM(Huh-7)-CM. (B) VEGF concentration of M0-CM, TAM(Huh-7)-CM and TAM(HepG2)-CM. The data are presented as the mean \pm SD. ${ }^{* * * *} \mathrm{P}<0.001$ (one-way ANOVA with the Tukey-Kramer test). TAMs, tumor-associated macrophages; VEGF, vascular endothelial growth factor; CM, conditioned media.

on cancer cells, the Huh-7 and HepG2 cells were cultured with TAM-CM or M0-CM, with the addition of VEGF antibody (Fig. S3A). The effects of TAM-CM on the Huh-7 and HepG2 cell proliferation and migration activities were attenuated at almost the same levels with M0-CM upon VEGF inhibition $(\mathrm{P}<0.05$, Fig. S3B-D). These results indicated that VEGF secreted by TAMs may promote cancer cell malignancy, further supporting its critical role in the TME.

VEGF inhibition induces the functional attenuation of TAMs without affecting M2 polarization. To clarify the critical role of VEGF in the interaction of TAMs and cancer cells, the effects of VEGF secreted by cancer cells on TAM polarization and function were investigated. VEGF secretion from cancer cells was confirmed (Fig. 2A), as well as VEGFR2 expression upregulation in TAMs from the Huh-7 and HepG2 cells, as compared its expression in M0 macrophages $(\mathrm{P}=0.04$ and $\mathrm{P}<0.001$, respectively, Fig. $2 \mathrm{~B})$. In order to investigate whether VEGF is involved in the establishment of TAM function during M2 polarization, M0 macrophages were cultured in cancer cell-CM in the presence or absence of VEGF antibody for $48 \mathrm{~h}$ (Fig. 3A). TAMs induced in the presence of the VEGF antibody (modified TAMs) displayed M2-like spindle-shaped morphological changes (data not shown), indicating that the inhibition of VEGF during M2 polarization did not affect TAM morphology. Likewise, modified TAMs exhibited similar mRNA expression levels of CD163 and CD206 as TAMs stimulated by cancer cell-CM without anti-VEGF antibody. These findings revealed that the inhibition of VEGF secreted by cancer cells did not affect the upregulation of the expression of CD163 and CD206, which are M2 macrophage markers (Fig. 3B). However, modified TAM-CM exerted weaker effects on cancer cell proliferation and migration compared with TAM-CM ( $\mathrm{P}<0.05$, Fig. 4A-C). VEGF secretion by modified TAMs was then investigated and it was revealed that modified TAMs displayed a significantly reduced VEGF secretion compared with TAMs $(\mathrm{P}<0.001$, Fig. 5A). Additionally, the secretion of MMP-9, which has been reported as one of the major factors secreted by TAMs to be positively correlated with VEGF secretion (25), 

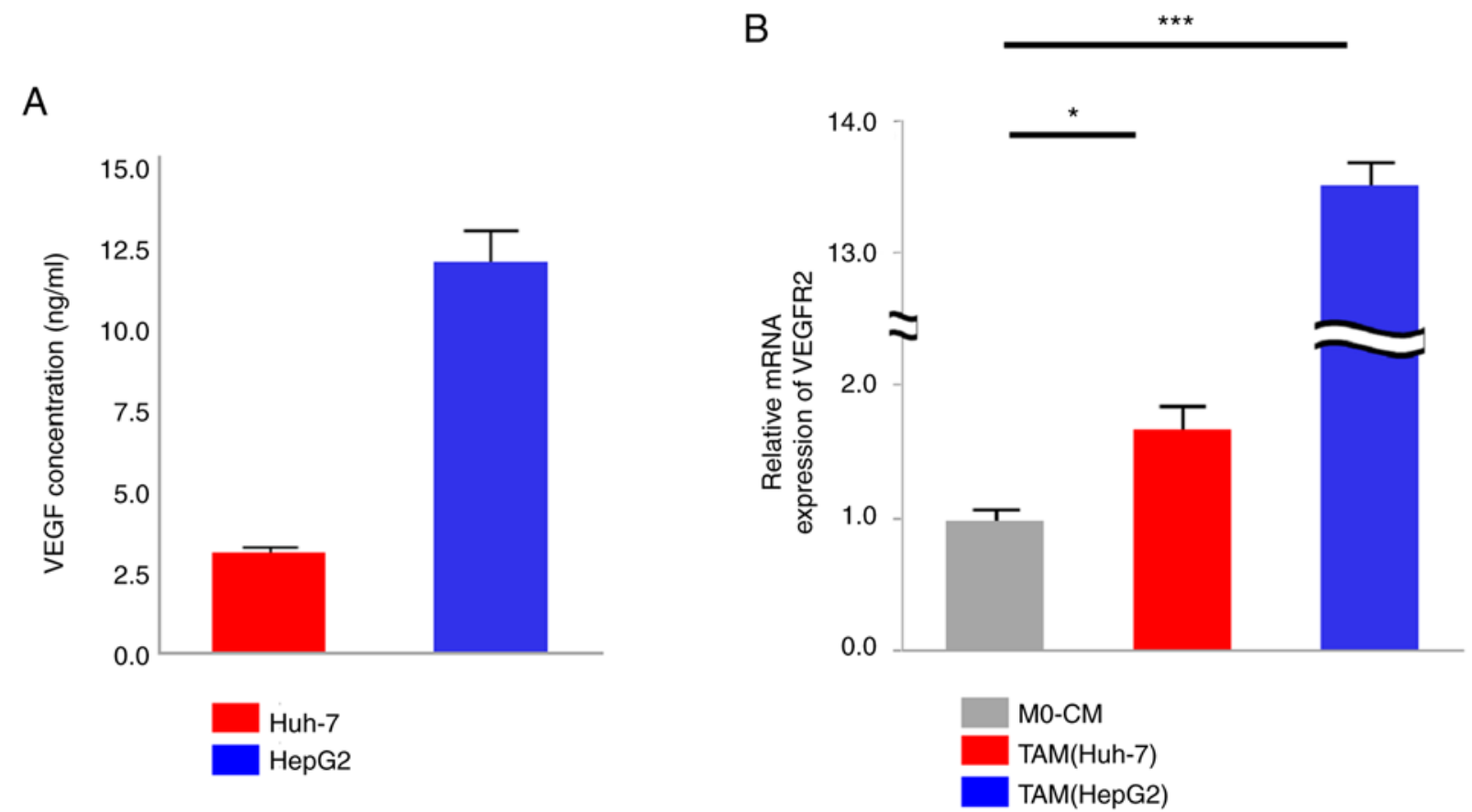

Figure 2. TAMs receive VEGF signals from cancer cells. (A) VEGF secretion by cancer cells (Huh-7, HepG2). (B) VEGFR2 mRNA expression in TAMs The data are presented as the mean $\pm \mathrm{SD} .{ }^{*} \mathrm{P}<0.05 ;{ }^{* * * *} \mathrm{P}<0.001$ (one-way ANOVA with the Tukey-Kramer test). VEGFR2, vascular endothelial growth factor receptor 2; TAMs, tumor-associated macrophages; VEGF, vascular endothelial growth factor.

was increased in TAMs and decreased in modified TAMs $(\mathrm{P}<0.05$, Fig. 5B).

Subsequently, the signaling pathways associated with VEGF secretion were examined in TAMs and modified TAMs. VEGFR2 mRNA expression was significantly downregulated in modified TAM(Huh-7) compared with the levels in TAM(Huh-7) $(\mathrm{P}=0.001$, Fig. 5C). The p-Akt and $\mathrm{p}$-mTOR levels were also decreased in the modified TAM(Huh-7) compared with levels in TAM(Huh-7), as revealed using western blot analysis $(\mathrm{P}<0.001$, Fig. 5D). These results suggested that VEGF inhibition during $\mathrm{M} 2$ polarization resulted in a decreased VEGF secretion in modified TAMs via the inactivation of the VEGFR2/Akt/mTOR pathway.

Modified TAMs have a reduced ability to upregulate PD-L1 expression. Several studies have previously reported that M2 macrophages may enhance PD-L1 expression in lung cancer, pancreatic cancer, and liver cancer (26-28). Thus, in the present study, Huh-7 cells were cultured with TAM(Huh-7)-CM or modified TAM(Huh-7)-CM for $48 \mathrm{~h}$, and PD-L1 mRNA expression in cancer cells was examined using RT-qPCR. The results demonstrated that TAM(Huh-7)-CM increased the mRNA expression of PD-L1 in the Huh-7 cells; however, modified TAM(Huh-7)-CM had no effect compared with M0-CM (Fig. 6A). Another study previously reported that TAMs not only promoted PD-L1 expression in cancer cells, but also expressed PD-L1 (29). Therefore, in the present study, PD-L1 mRNA expression was examined and its upregulation in TAMs was detected, while a weaker upregulation was detected in modified TAM(Huh-7) compared with TAM(Huh-7) (Fig. 6B). These findings illustrated that VEGF inhibition attenuated the expression of PD-L1 and the tumor immunosuppression function in TAMs.

\section{Discussion}

In the present study, it was demonstrated that the inhibition of VEGF secreted by cancer cells did not alter the M2 polarization of macrophages in the TME; however, the tumor-promoting characteristics of TAMs were attenuated though the suppression of VEGF secretion in TAMs. TAMs induced in a VEGF-depleted environment exhibited reduced activation of the Akt/mTOR signaling pathway and decreased secretion of humoral factors, including VEGF and MMP-9. Furthermore, the increased PD-L1 expression in cancer cells was not achieved in the presence of TAMs induced in a VEGF-depleted environment, with the PD-L1 expression of these modified TAMs also being suppressed.

Furthermore, the effect of VEGF inhibition on the M2 polarization of macrophages in the TME was investigated. TAMs are mainly M2 macrophages that are activated by tumor-derived IL-4, IL-13, IL-10, macrophage colony-stimulating factor and lactic acid in the TME. M2 macrophages produce anti-inflammatory cytokines, such as IL-10, IL-13 and transforming growth factor- $\beta$ to promote tumor development and growth (30-32). It has been previously reported by the authors that TAMs and cancer cells may interact via the Nrf2 pathway, VEGF secretion by TAMs may enhance EMT of cancer cells and that lactic acid secreted by cancer cells may polarize macrophages toward the M2 phenotype (18). However, contrary to the current expectations, the present study demonstrated that VEGF inhibition by VEGF antibody in the cancer cell-CM did not contribute to M2 macrophage polarization inhibition.

The function of TAMs stimulated by cancer cell-CM in a VEGF-depleted environment was further investigated using VEGF antibody. Previous studies have demonstrated that 

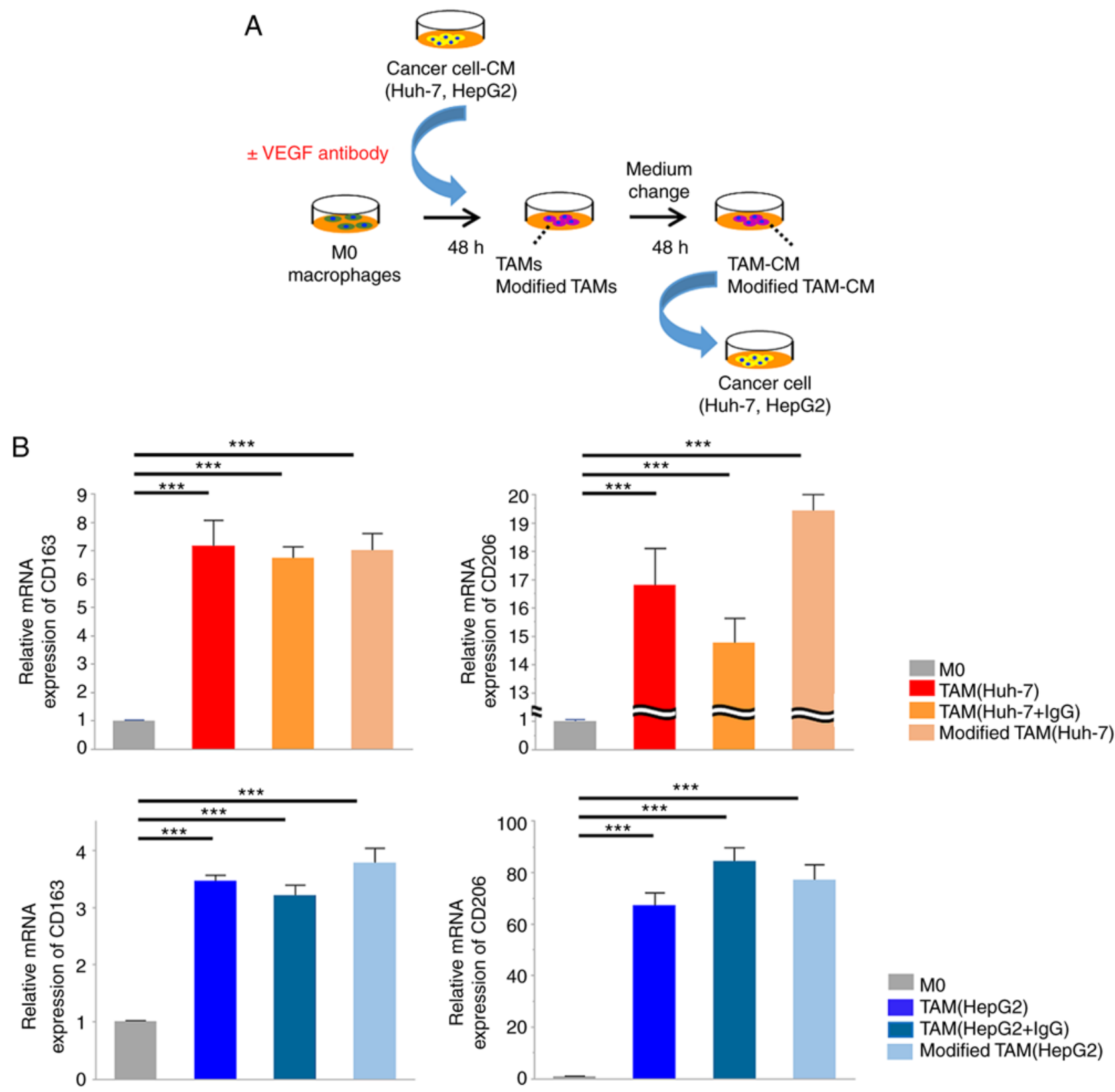

Figure 3. VEGF inhibition does not affect macrophage M2 polarization. (A) Experimental schematic diagram of the examination of the VEGF inhibitor effects on TAMs during M2 polarization. The same concentration of IgG was used as a control. (B) M2 marker mRNA expression in TAMs and modified TAMs. The data are presented as the mean $\pm \mathrm{SD} .{ }^{* * *} \mathrm{P}<0.001$ (one-way ANOVA with the Tukey-Kramer test). VEGF, vascular endothelial growth factor; TAMs, Tumor-associated macrophages.

TAMs may contribute to tumor neovascularization through the upregulation of VEGF secretion, and the MMP-induced degradation of extracellular matrix surrounding cancer cells, resulting in the release of heparin-bound growth factors, including VEGF, to further support angiogenesis and tumor progression $(33,34)$. The results of the present study indicated that modified TAMs, which were induced in a VEGF-depleted environment, exhibited a weaker ability to promote the proliferation and migration of cancer cells due to a reduction in the VEGF secretion level. VEGFR2 is expressed on macrophages, and its expression is upregulated on M2 macrophages (35). VEGF secreted by TAMs has been reported to function simultaneously in a paracrine and autocrine manner through the VEGFR2 signaling pathway in TAMs, as also observed in cancer cells. Recently, several studies have reported that VEGFR2 expression on M2 TAMs plays a crucial role in tumor immune tolerance within the TME $(19,35)$. Other studies have previously suggested that humoral factor secretion by M2 macrophages may be regulated via the VEGF/VEGFR2 signaling pathway (36-38). The present study confirmed that VEGF depletion suppressed Akt/mTOR pathway activation, which is a main downstream pathway of VEGF/VEGFR2 signaling, in modified TAMs. The inactivation of the Akt/mTOR pathway has been previously reported to reduce VEGFR2 expression in glioma cells (39), and the inhibition of the mTOR pathway has been reported to downregulate the production of VEGF and MMP-9 in macrophages and cancer cells $(40,41)$. These reports are in support of the present results, 
A

Huh-7

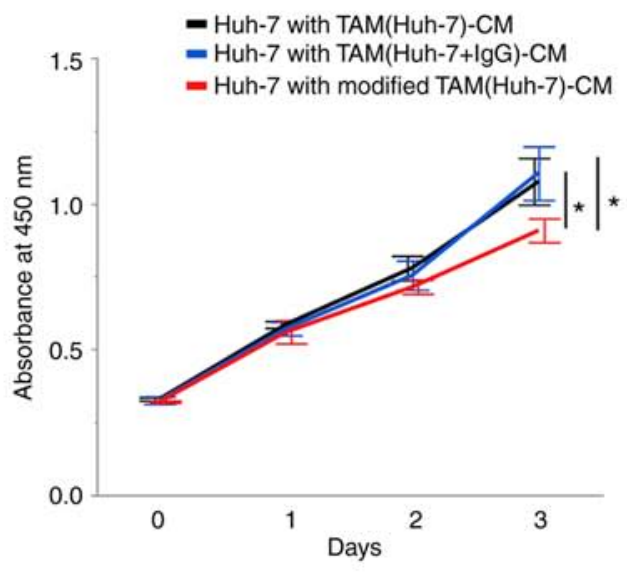

B
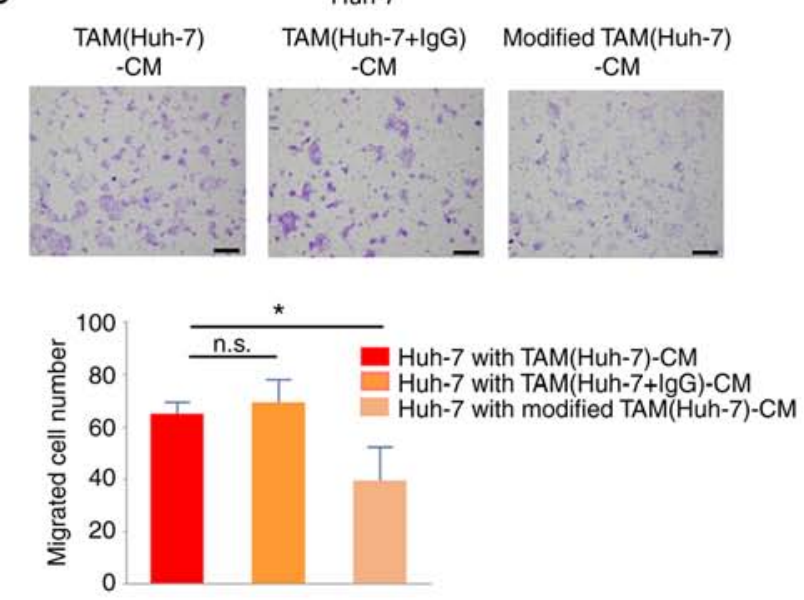

C

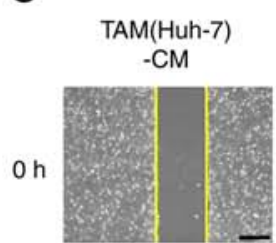

Huh-7

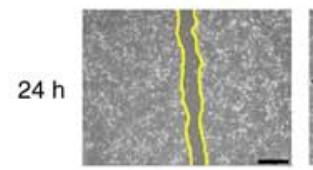

TAM(Huh-7+lgG) $-\mathrm{CM}$

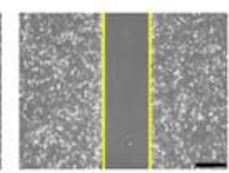

Modified TAM(Huh-7)
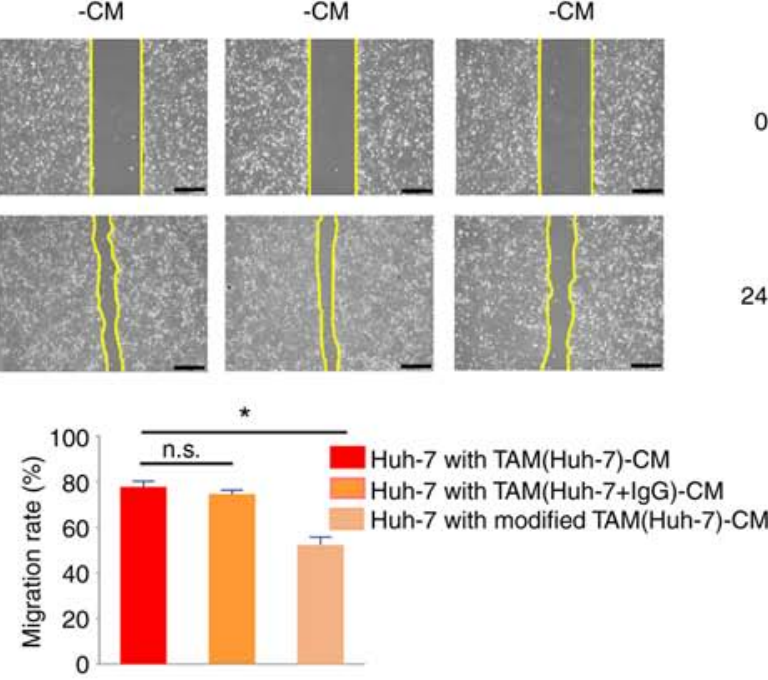

HepG2
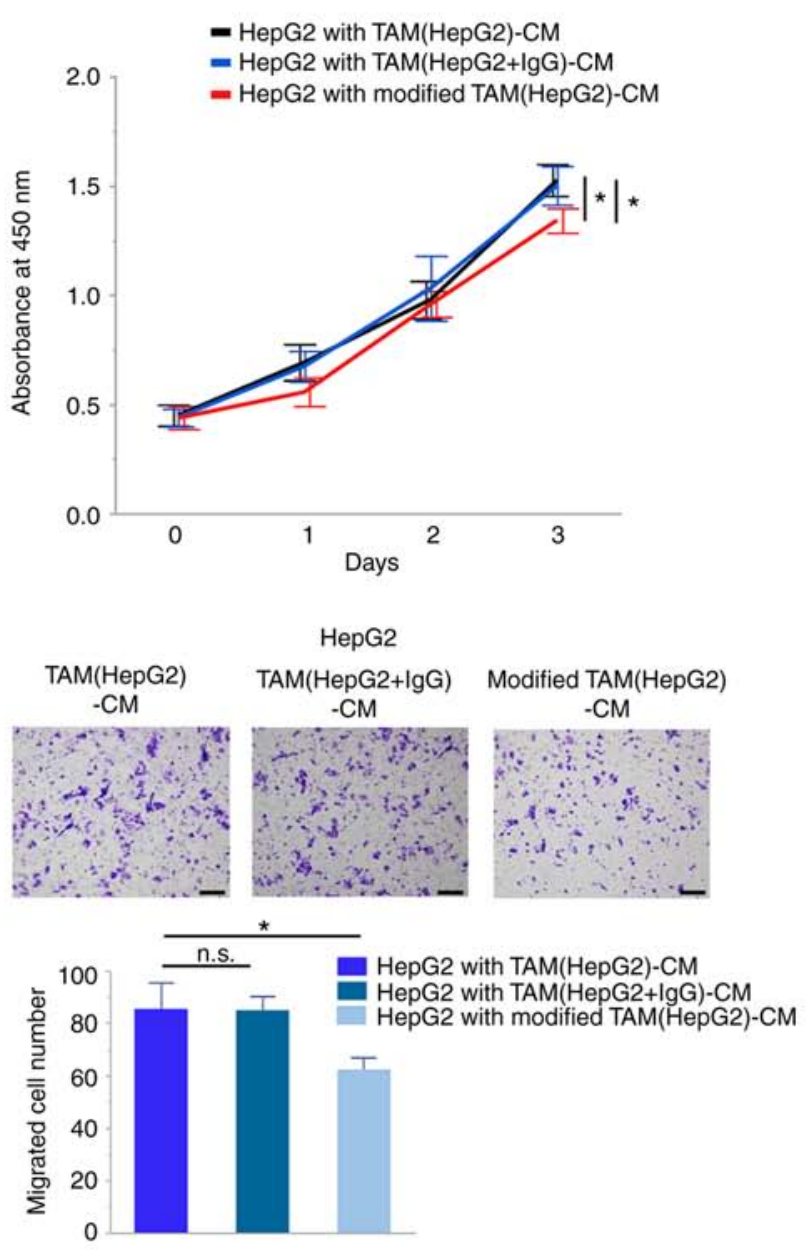

HepG2

TAM(HepG2) TAM(HepG2+lgG) Modified TAM(HepG2) $-\mathrm{CM}$
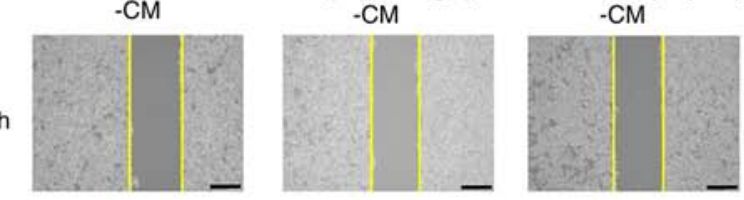

$24 \mathrm{~h}$
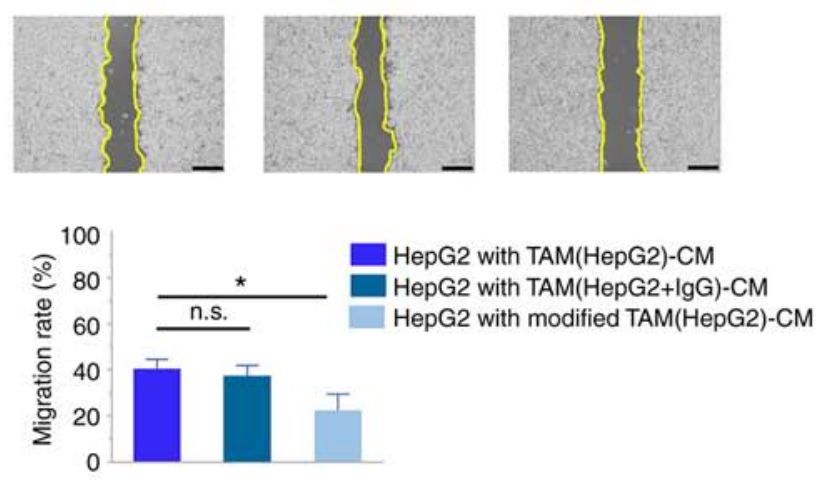

Figure 4. VEGF inhibition reduces the malignant potential of TAMs. (A) Proliferation assay of Huh-7 and HepG2 cells cultured with modified TAM-CM. (B) Transwell migration assay (scale bar, $100 \mu \mathrm{m}$; magnification, x200) of Huh-7 and HepG2 cells cultured with modified TAM-CM. (C) Wound-healing assay (scale bar, $500 \mu \mathrm{m}$; magnification, $\mathrm{x} 40$ ) of Huh-7 and HepG2 cells cultured with modified TAM-CM. The data are presented as the mean \pm SD. ${ }^{*} \mathrm{P}<0.05$; n.s., not significant (one-way ANOVA with the Tukey-Kramer test). VEGF, vascular endothelial growth factor; TAMs, tumor-associated macrophages; $\mathrm{CM}$, conditioned medium.

concerning the modified TAMs demonstrating attenuated cytokine secretion via inactivation of VEGFR2/Akt/mTOR pathway. This finding suggests that the inhibition of autocrine and paracrine VEGF secretion by TAMs and cancer cells may represent a treatment strategy for inhibiting the TME, thereby suppressing tumor progression. 

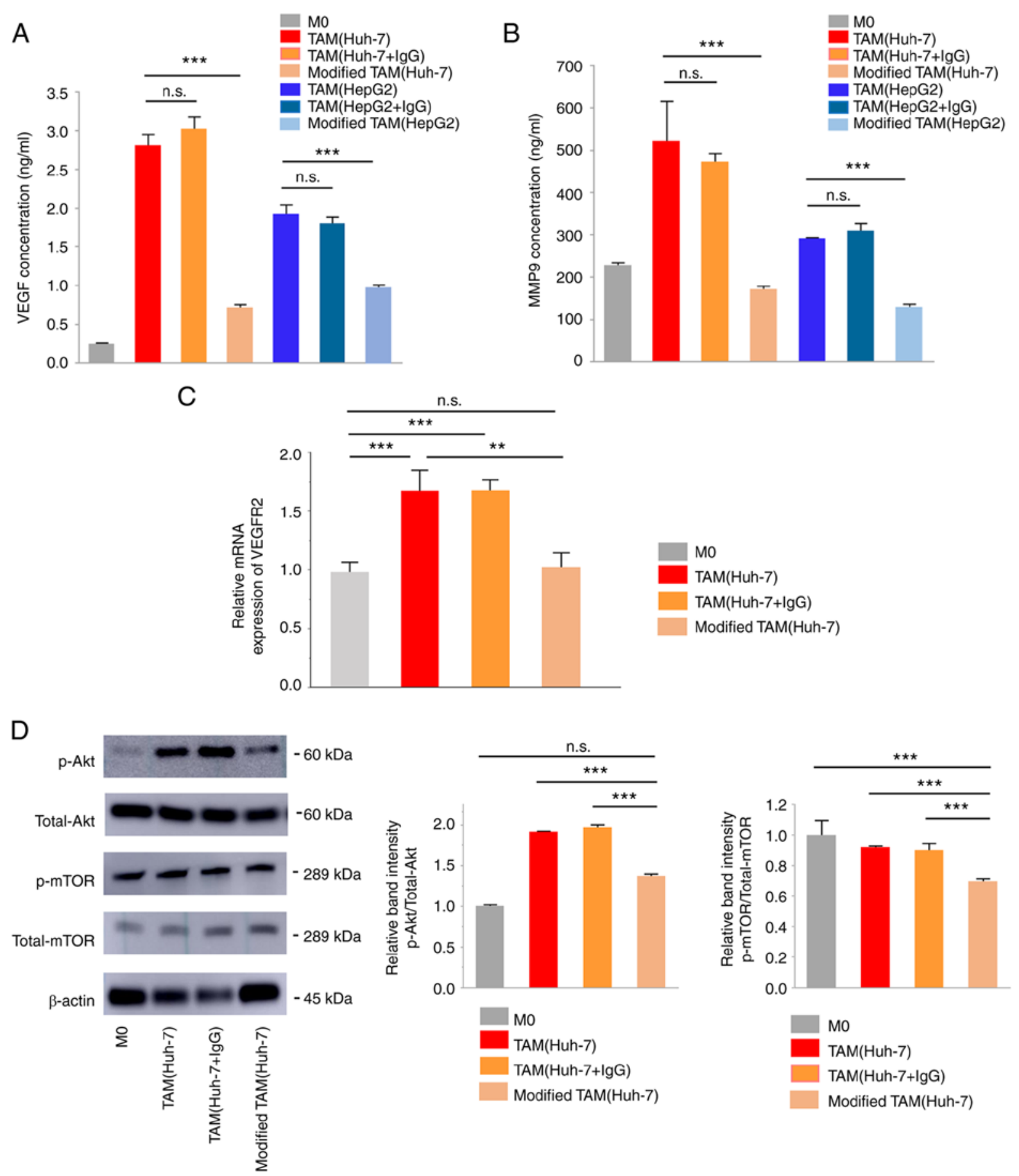

Figure 5. VEGF inhibition suppresses the ability of TAMs to secrete cytokines via the VEGFR2/Akt/mTOR pathway. (A) VEGF secretion by TAMs and modified TAMs. (B) MMP-9 secretion by TAMs and modified TAMs. (C) VEGFR2 expression in TAMs and modified TAMs. (D) Western blots of Akt/mTOR signaling components. The data are presented as the mean $\pm \mathrm{SD} .{ }^{* *} \mathrm{P}<0.01 ;{ }^{* * *} \mathrm{P}<0.001 ;$ n.s., not significant (one-way ANOVA with the Tukey-Kramer test). VEGF, vascular endothelial growth factor; TAMs, tumor-associated macrophages; MMP-9, matrix metalloproteinase-9.

Finally, the present study investigated whether the VEGF-depleted environment was associated with tumor immune tolerance via PD-L1 expression in cancer cells and TAMs. Shima et al (28) reported that macrophages significantly expressed PD-L1 during TAM-like M2 differentiation, and TGF- $\beta$ produced by TAMs induced PD-L1 expression in lung cancer cells. Yao et al (42) reported that PD-L1 expression by macrophages was positively regulated by the PI3K/Akt signaling pathway, in support of the current results. Another study previously reported that PD-L1 expression by liver cancer cells was regulated by the Nrf2 pathway (43), and a previous report by the authors previously revealed that VEGF secreted by TAMs may activate the Nrf2 pathway in liver cancer cells (18). Therefore, VEGF inhibition can suppress the VEGF autocrine loop in macrophages and reduce PD-L1 expression in cancer cells through the inactivation of the AKT/mTOR pathway in TAMs, possibly due to the suppression of the Nrf2 pathway in cancer cells.

Recent large-scale randomized clinical trials have demonstrated that the concomitant use of anti-VEGF treatment 
A

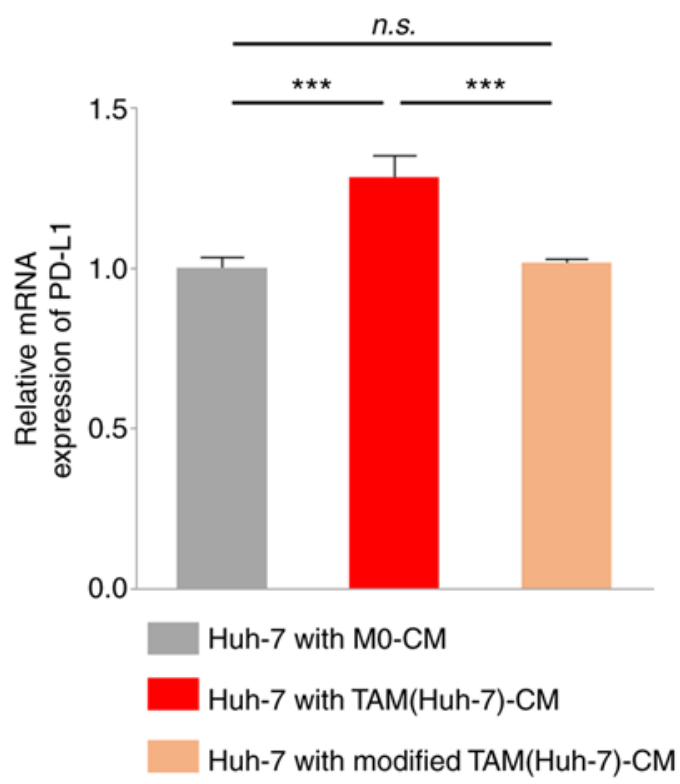

B

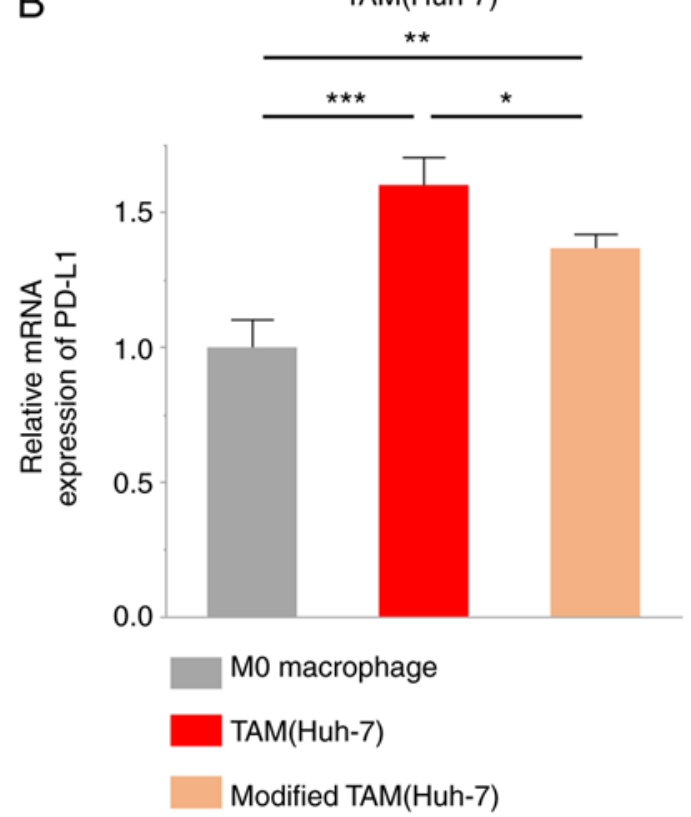

Figure 6. VEGF inhibition decreases PD-L1 expression in TAMs and cancer cells. (A) PD-L1 expression in cancer cells. (B) PD-L1 expression in TAMs and modified TAMs. The data are presented as the mean $\pm \mathrm{SD} .{ }^{*} \mathrm{P}<0.05 ;{ }^{* *} \mathrm{P}<0.01 ;{ }^{* * *} \mathrm{P}<0.001$; n.s., not significant (one-way ANOVA with the Tukey-Kramer test). VEGF, vascular endothelial growth factor; PD-L1, programmed death-ligand 1; TAMs, tumor-associated macrophages.

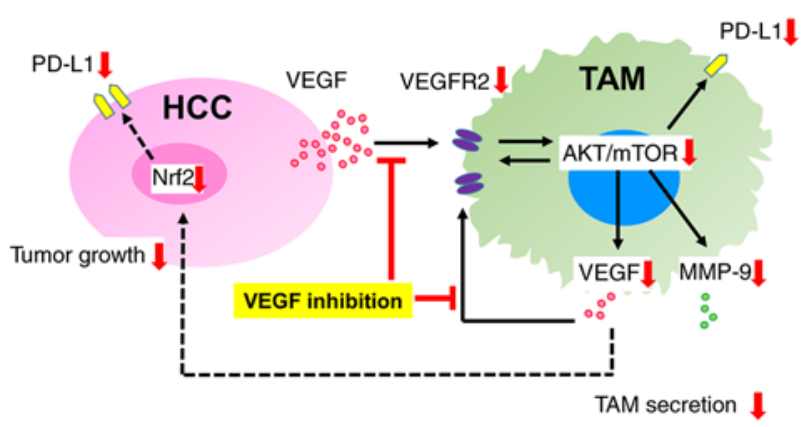

Figure 7. Proposed mechanisms of VEGF inhibition in the TME and its effects on TAMs. VEGF inhibition in the TME did not affect the M2 polarization of macrophages. TAMs stimulated in a VEGF-depleted environment displayed reduced M2 characteristics, such as the secretion of VEGF and other cytokines including MMP-9 and reduced immune tolerance of cancer cells through PD-L1 in TAMs and cancer cells. This may be as VEGF depletion induces the suppression of VEGF signaling pathways, including the VEGFR2/Akt/mTOR pathway in macrophages. VEGF, vascular endothelial growth factor; TME, tumor microenvironment; TAMs, tumor-associated macrophages; MMP-9, matrix metalloproteinase-9; PD-L1, programmed death-ligand 1.

enhances the efficacy of anti-PD-L1 therapy in advanced liver cancer, and this strategy has already been clinically introduced globally. The results of clinical trials have demonstrated that treatment with the combination of anti-PD-L1 and anti-VEGF therapy is effective due to their synergistic effects on tumor growth, as well as due to their ability to reprogram the immunosuppressive environment to enhance anti-tumor immune responses $(21,22)$. Although the effects of combination therapy have been clinically confirmed, only a limited number of studies support the co-association between VEGF and PD-L1. Schmidinger (44) reported that PD-L1 expression, as detected by immunohistochemistry, was associated with VEGF expression, which reflected poor pathological features in patients with clear cell renal cell carcinoma. The findings of the present study suggested that the TAMs induced in a VEGF-depleted environment had a weaker ability to promote tumor progression and that VEGF inhibition in the TME regulates PD-L1 expression in TAMs and cancer cells (Fig. 7). This concept could help clarify the mechanisms of the effects of the combined anti-PD-L1 and anti-VEGF therapy.

The present study has several limitations. Firstly, the results were based on in vitro experiments using VEGF antibody. To further investigate the effects of the inhibition of the VEGF signal pathway in TAMs, genetic modification technology targeting VEGF, including shRNA are required, as well as experiments using a liver cancer animal model.

In conclusion, the importance of VEGF signaling for the malignant potential of TAMs in the TME of liver cancer was demonstrated in the present study. VEGF inhibitors may not affect M2 polarization; however, VEGF inhibition impedes tumor growth and attenuates TAM function. These effects may suppress tumor progression and reduce tumor immune escape through the inactivation of the VEGFR2/AKT/mTOR pathway. Therefore, the present study revealed that VEGF inhibition can lead to the functional deficiency of TAMs, in support of the potential efficacy of the combined anti-VEGF and anti-PD-L1 treatment.

\section{Acknowledgements}

Not applicable.

\section{Funding}

The present study was supported in part by the Research Program on Hepatitis from the Japanese Foundation for Multidisciplinary Treatment of Cancer, the Japan Agency for 
Medical Research and Development (grant nos. JP19fk0210048 and JP20fk0210048), and Grants-in-Aid for Scientific Research (grant nos. 20K08957 and 18K02871). This study was also funded by Taiho Pharmaceutical Co., Ltd. (Tokyo, Japan).

\section{Availability of data and materials}

The datasets used and/or analyzed during the current study are available from the corresponding author on reasonable request.

\section{Authors' contributions}

SO, KT, YM and MS designed the experiments. SO, SYamas, $\mathrm{KM}$ and HT performed the experiments and collected the data. YS, SYamad, TI and SI analyzed and interpreted the data. SO drafted the manuscript. YM and MS revised the paper critically for important intellectual content. SO and YM confirmed the authenticity of all the raw data. SO and YM agree to be accountable for all aspects of the work in ensuring that questions related to the accuracy or integrity of any part of the work are appropriately investigated and resolved. All the authors have read and approved the final version of the manuscript for publication.

\section{Ethics approval and consent to participate}

Not applicable.

\section{Patient consent for publication}

Not applicable.

\section{Competing interests}

All the authors declare that they have no competing interests.

\section{References}

1. Casey SC, Li Y, Fan AC and Felsher DW: Oncogene withdrawal engages the immune system to induce sustained cancer regression. J Immunother Cancer 2: 24, 2014.

2. Kenny PA, Lee GY and Bissell MJ: Targeting the tumor microenvironment. Front Biosci 12: 3468-3474, 2007.

3. Zhou K, Cheng T, Zhan J, Peng X, Zhang Y, Wen J, Chen X and Ying M: Targeting tumor-associated macrophages in the tumor microenvironment. Oncol Lett 20: 234, 2020.

4. Mantovani A, Sozzani S, Locati M, Allavena P and Sica A: Macrophage polarization: Tumor-associated macrophages as a paradigm for polarized M2 mononuclear phagocytes. Trends Immunol 23: 549-555, 2002.

5. Lan C, Huang X, Lin S, Huang H, Cai Q, Wan T, Lu J and Liu J: Expression of M2-polarized macrophages is associated with poor prognosis for advanced epithelial ovarian cancer. Technol Cancer Res Treat 12: 259-267, 2013.

6. Kurahara H, Shinchi H, Mataki Y, Maemura K, Noma H, Kubo F, Sakoda M, Ueno S, Natsugoe S and Takao S: Significance of M2-polarized tumor-associated macrophage in pancreatic cancer. J Surg Res 167: e211-e219, 2011.

7. Zhang B, Yao G, Zhang Y, Gao J, Yang B, Rao Z and Gao J: M2-polarized tumor-associated macrophages are associated with poor prognoses resulting from accelerated lymphangiogenesis in lung adenocarcinoma. Clinics (Sao Paulo) 66: 1879-1886, 2011.

8. Chanmee T, Ontong P, Konno K and Itano N: Tumor-associated macrophages as major players in the tumor microenvironment. Cancers (Basel) 6: 1670-1690, 2014.

9. Qian BZ and Pollard JW: Macrophage diversity enhances tumor progression and metastasis. Cell 141: 39-51, 2010.
10. Condeelis J and Pollard JW: Macrophages: Obligate partners for tumor cell migration, invasion, and metastasis. Cell 124: 263-266, 2006.

11. Pollard JW: Tumour-educated macrophages promote tumour progression and metastasis. Nat Rev Cancer 4: 71-78, 2004.

12. Valković T, Dobrila F, Melato M, Sasso F, Rizzardi C and Jonjić N: Correlation between vascular endothelial growth factor, angiogenesis, and tumor-associated macrophages in invasive ductal breast carcinoma. Virchows Arch 440: 583-588, 2002.

13. Shieh YS, Hung YJ, Hsieh CB, Chen JS, Chou KC and Liu SY: Tumor-associated macrophage correlated with angiogenesis and progression of mucoepidermoid carcinoma of salivary glands. Ann Surg Oncol 16: 751-760, 2009.

14. Deryugina EI and Quigley JP: Tumor angiogenesis: MMPmediated induction of intravasation- and metastasis-sustaining neovasculature. Matrix Biol 44-46: 94-112, 2015.

15. Lin Y, Zhai E, Liao B, Xu L, Zhang X, Peng S, He Y, Cai S, Zeng $Z$ and Chen $M$ : Autocrine VEGF signaling promotes cell proliferation through a PLC-dependent pathway and modulates Apatinib treatment efficacy in gastric cancer. Oncotarget 8: 11990-12002, 2017.

16. Capece D, Fischietti M, Verzella D, Gaggiano A, Cicciarelli G, Tessitore A, Zazzeroni $\mathrm{F}$ and Alesse $\mathrm{E}$ : The inflammatory microenvironment in hepatocellular carcinoma: A pivotal role for tumor-associated macrophages. Biomed Res Int 2013: 187204, 2013.

17. Wu K, Lin K, Li X, Yuan X, Xu P, Ni P and Xu D: Redefining tumor-associated macrophage subpopulations and functions in the tumor microenvironment. Front Immunol 11: 1731, 2020.

18. Feng R, Morine Y, Ikemoto T, Imura S, Iwahashi S, Saito Y and Shimada M: Nrf2 activation drive macrophages polarization and cancer cell epithelial-mesenchymal transition during interaction. Cell Commun Signal 16: 54, 2018.

19. Wheeler KC, Jena MK, Pradhan BS, Nayak N, Das S, Hsu CD, Wheeler DS, Chen K and Nayak NR: VEGF may contribute to macrophage recruitment and M2 polarization in the decidua. PLoS One 13: e0191040, 2018.

20. Linde N, Lederle W, Depner S, van Rooijen N, Gutschalk CM and Mueller MM: Vascular endothelial growth factor-induced skin carcinogenesis depends on recruitment and alternative activation of macrophages. J Pathol 227: 17-28, 2012.

21. Finn RS, Qin S, Ikeda M, Galle PR, Ducreux M, Kim TY, Kudo M, Breder V, Merle P, Kaseb AO, et al: Atezolizumab plus bevacizumab in unresectable hepatocellular carcinoma. N Engl J Med 382: 1894-1905, 2020.

22. Cheng AL, Qin S, Ikeda M, Galle P, Ducreux M, Zhu A, Kim TY, Kudo M, Breder V, Merle P, et al: IMbrave150: Efficacy and safety results from a ph III study evaluating atezolizumab (atezo) + bevacizumab (bev) vs sorafenib (Sor) as first treatment (tx) for patients (pts) with unresectable hepatocellular carcinoma (HCC). Ann Oncol 30: ix186-ix187, 2019.

23. Livak KJ and Schmittgen TD: Analysis of relative gene expression data using real-time quantitative PCR and the 2(-Delta Delta C(T)) method. Methods 25: 402-408, 2001.

24. Yoshimoto T,Morine Y, Takasu C,Feng R,Ikemoto T, YoshikawaK, Iwahashi S, Saito Y, Kashihara H, Akutagawa M, et al: Blue light-emitting diodes induce autophagy in colon cancer cells by Opsin 3. Ann Gastroenterol Surg 2: 154-161, 2018.

25. Riabov V, Gudima A, Wang N, Mickley A, Orekhov A and Kzhyshkowska J: Role of tumor associated macrophages in tumor angiogenesis and lymphangiogenesis. Front Physiol 5: 75, 2014.

26. Tsukamoto M, Imai K, Ishimoto T, Komohara Y, Yamashita YI Nakagawa S, Umezaki N, Yamao T, Kitano Y, Miyata T, et al: PD-L1 expression enhancement by infiltrating macrophagederived tumor necrosis factor- $\alpha$ leads to poor pancreatic cancer prognosis. Cancer Sci 110: 310-320, 2019.

27. Wei Y, Zhao Q, Gao Z, Lao XM, Lin WM, Chen DP, Mu M, Huang CX, Liu ZY, Li B, et al: The local immune landscape determines tumor PD-L1 heterogeneity and sensitivity to therapy. J Clin Invest 129: 3347-3360, 2019.

28. Shima T, Shimoda M, Shigenobu T, Ohtsuka T, Nishimura T, Emoto K, Hayashi Y, Iwasaki T, Abe T, Asamura H and Kanai Y: Infiltration of tumor-associated macrophages is involved in tumor programmed death-ligand 1 expression in early lung adenocarcinoma. Cancer Sci 111: 727-738, 2020.

29. Lai YS, Wahyuningtyas R, Aui SP and Chang KT: Autocrine VEGF signalling on M2 macrophages regulates PD-L1 expression for immunomodulation of T cells. J Cell Mol Med 23: 1257-1267, 2019.

30. Sica A and Mantovani A: Macrophage plasticity and polarization: In vivo veritas. J Clin Invest 122: 787-795, 2012. 
31. Mantovani A, Biswas SK, Galdiero MR, Sica A and Locati M Macrophage plasticity and polarization in tissue repair and remodelling. J Pathol 229: 176-185, 2013.

32. Petty AJ and Yang Y: Tumor-associated macrophages: Implications in cancer immunotherapy. Immunotherapy 9: 289-302, 2017.

33. Hughes R, Qian BZ, Rowan C, Muthana M, Keklikoglou I, Olson OC, Tazzyman S, Danson S, Addison C, Clemons M, et al Perivascular M2 macrophages stimulate tumor relapse after chemotherapy. Cancer Res 75: 3479-3491, 2015.

34. Osterberg N, Ferrara N, Vacher J, Gaedicke S, Niedermann G, Weyerbrock A, Doostkam S, Schaefer HE, Plate KH and Machein MR: Decrease of VEGF-A in myeloid cells attenuates glioma progression and prolongs survival in an experimental glioma model. Neuro Oncol 18: 939-949, 2016.

35. Min AKT, Mimura K, Nakajima S, Okayama H, Saito K, Sakamoto W, Fujita S, Endo H, Saito M, Saze Z, et al: Therapeutic potential of anti-VEGF receptor 2 therapy targeting for M2-tumor-associated macrophages in colorectal cancer. Cancer Immunol Immunother 70: 289-298, 2021.

36. Chu M, Xu L, Zhang MB, Chu ZY and Wang YD: Role of baicalin in anti-influenza virus $A$ as a potent inducer of IFN-gamma. Biomed Res Int 2015: 263630, 2015.

37. Yu FY, Huang SG, Zhang HY, Chi HG, Zou Y, Lu RX and Zheng XB: Effect of baicalin on signal transduction and activating transcription factor expression in ulcerative colitis patients. Zhongguo Zhong Xi Yi Jie He Za Zhi 35: 419-424, 2015 (In Chinese).

38. Zhang CL, Zhang S, He WX, Lu JL, Xu YJ, Yang JY and Liu D: Baicalin may alleviate inflammatory infiltration in dextran sodium sulfate-induced chronic ulcerative colitis via inhibiting IL-33 expression. Life Sci 186: 125-132, 2017.
39. Kessler T, Sahm F, Blaes J, Osswald M, Rübmann P, Milford D, Urban S, Jestaedt L, Heiland S, Bendszus M, et al: Glioma cell VEGFR-2 confers resistance to chemotherapeutic and antiangiogenic treatments in PTEN-deficient glioblastoma. Oncotarget 6: 31050-31068, 2015.

40. Faes S, Santoro T, Demartines N and Dormond O: Evolving significance and future relevance of anti-angiogenic activity of mTOR inhibitors in cancer therapy. Cancers (Basel) 9: 152, 2017.

41. Mercurio A, Lipscomb E and Bachelder R: Non-angiogenic functions of VEGF in breast cancer. J Mammary Gland Biol Neoplasia 10: 283-290, 2005.

42. Yao X, Tu Y, Xu Y, Guo Y, Yao F and Zhang X: Endoplasmic reticulum stress-induced exosomal miR-27a-3p promotes immune escape in breast cancer via regulating PD-L1 expression in macrophages. J Cell Mol Med 24: 9560-9573, 2020.

43. Li D, Sun FF, Wang D, Wang T, Peng JJ, Feng JQ, Li H, Wang C, Zhou DJ, Luo H, et al: Programmed death ligand-1 (PD-L1) regulated by NRF-2/MicroRNA-1 regulatory axis enhances drug resistance and promotes tumorigenic properties in sorafenib-resistant hepatoma cells. Oncol Res 28: 467-481, 2020.

44. Schmidinger M: Clinical decision-making for immunotherapy in metastatic renal cell carcinoma. Curr Opin Urol 28: 29-34, 2018.

This work is licensed under a Creative Commons Attribution-NonCommercial-NoDerivatives 4.0 International (CC BY-NC-ND 4.0) License. 